\title{
Colouring planar mixed hypergraphs
}

\author{
André Kündgen* \\ The Fields Institute \\ 222 College St. \\ Toronto, Ont. M5T 3J1 \\ Canada \\ kundgen@member.ams.org
}

\author{
Eric Mendelsohn ${ }^{\dagger}$ \\ Department of Mathematics \\ University of Toronto \\ 100 St. George St., Toronto, Ont., \\ Canada \\ mendelso@math.utoronto.ca
}

\author{
Vitaly Voloshin ${ }^{\ddagger}$ \\ Institute of Mathematics and Informatics \\ Moldavian Academy of Sciences \\ Academiei, 5, Chişinău, MD-2028 \\ Moldova \\ voloshin@math.mldnet.com \\ Submitted: June 5, 2000; Accepted September 28, 2000
}

\begin{abstract}
A mixed hypergraph is a triple $\mathcal{H}=(V, \mathcal{C}, \mathcal{D})$ where $V$ is the vertex set and $\mathcal{C}$ and $\mathcal{D}$ are families of subsets of $V$, the $\mathcal{C}$-edges and $\mathcal{D}$-edges, respectively. A $k$-colouring of $\mathcal{H}$ is a mapping $c: V \rightarrow[k]$ such that each $\mathcal{C}$-edge has at least two vertices with a $\mathcal{C}$ ommon colour and each $\mathcal{D}$-edge has at least two vertices of $\mathcal{D}$ ifferent colours. $\mathcal{H}$ is called a planar mixed hypergraph if its bipartite representation is a planar graph. Classic graphs are the special case of mixed hypergraphs when $\mathcal{C}=\emptyset$ and all the $\mathcal{D}$-edges have size 2 , whereas in a bi-hypergraph $\mathcal{C}=\mathcal{D}$.

We investigate the colouring properties of planar mixed hypergraphs. Specifically, we show that maximal planar bi-hypergraphs are 2-colourable, find formulas for their chromatic polynomial and chromatic spectrum in terms of 2-factors in the dual, prove that their chromatic spectrum is gap-free and provide a sharp estimate on the maximum number of colours in a colouring.
\end{abstract}

\footnotetext{
*Supported by NSERC grant of Derek Corneil and the Fields Institute.

${ }^{\dagger}$ This research is supported by NSERC Canada OGP007621 and the Fields Institute.

$¥$ This research is supported by NSERC Canada OGP007621, OGP007268 and the Fields Institute.
} 
THE EleCtronic Journal of COMBinatorics 7 (2000), \#R60

Keywords: colourings of hypergraphs, mixed hypergraphs, planar graphs and hypergraphs, colourability, chromatic spectrum.

2000 Mathematics Subject Classification: 05C15.

\section{Introduction}

We use the standard graph and hypergraph terminology of Berge [1, 2] and the mixed hypergraph terminology from $[10,11,12,13]$. Following Berge $[1,2]$ a hypergraph is a pair $(V, \mathcal{E})$ where $V$ is the set of vertices and $\mathcal{E}$ is a family of subsets of $V$ (of size at least 2 ), the edges. We use this notation mainly when we discuss general properties. When we consider colouring properties specifically, we shall use the language of mixed hypergraphs.

A mixed hypergraph is a triple $\mathcal{H}=(V, \mathcal{C}, \mathcal{D})$ where $V$ is the vertex set, $|V|=n$, and $\mathcal{C}$ and $\mathcal{D}$ are families of subsets of $V$, the $\mathcal{C}$-edges and $\mathcal{D}$-edges.

A (proper) $k$-colouring of a mixed hypergraph is a mapping $c: V \rightarrow\{1,2, \ldots, k\}$ from the vertex set $V$ into a set of $k$ colours so that each $\mathcal{C}$-edge has at least two vertices with $\mathcal{C}$ ommon colour and each $\mathcal{D}$-edge has at least two vertices with $\mathcal{D}$ ifferent colours. A strict $k$-colouring is a proper colouring using all $k$ colours. If a mixed hypergraph $\mathcal{H}$ has at least one colouring, then $\mathcal{H}$ is called colourable. Otherwise $\mathcal{H}$ is called uncolourable. Let $P(\mathcal{H}, k)$ denote the number of proper $k$-colorings of $\mathcal{H}$. It can be seen that $P(\mathcal{H}, k)$ is a polynomial in $k$, known as the chromatic polynomial of $\mathcal{H}$. By $c(v)$ we denote the colour of vertex $v \in V$ in the colouring $c$. A set of vertices is monochromatic in a colouring if all the vertices of the set have the same colour. Similarly a set is polychromatic if no two vertices in it have the same colour. Thus in a proper colouring $\mathcal{C}$-edges must be non-polychromatic subsets, and $\mathcal{D}$-edges non-monochromatic subsets of vertices. If $\mathcal{H}$ is colourable then the minimum number of colours over all colourings is the lower chromatic number $\chi(\mathcal{H})$. The maximum number of colours in all strict colourings of $\mathcal{H}$ is its upper chromatic number $\bar{\chi}(\mathcal{H})$.

For each $k, 1 \leq k \leq n$, let $r_{k}$ be the number of partitions of the vertex set into $k$ nonempty parts (colour classes) such that the colouring constraint is satisfied on each $\mathcal{C}$ and $\mathcal{D}$-edge. We call these partitions feasible. Thus $r_{k}$ is the number of different strict $k$-colourings of $\mathcal{H}$ if we disregard permutations of colours. The vector

$$
R(\mathcal{H})=\left(r_{1}, \ldots, r_{n}\right)=\left(0, \ldots, 0, r_{\chi(\mathcal{H})}, \ldots, r_{\bar{\chi}(\mathcal{H})}, 0, \ldots, 0\right)
$$

is the chromatic spectrum of $\mathcal{H}$.

The set of values $k$ such that $\mathcal{H}$ has a strict $k$-colouring is the feasible set of $\mathcal{H}$; this is the set of indices $i$ such that $r_{i}>0$. A mixed hypergraph has a gap at $k$ if its feasible set contains elements larger and smaller than $k$ but omits $k$. A mixed hypergraph $\mathcal{H}$ is called uniquely colourable [11] if it has precisely one feasible partition.

A mixed hypergraph $\mathcal{H}=(V, \mathcal{C}, \mathcal{D})$ is called reduced if every $\mathcal{C}$-edge is of size at least 3 , every $\mathcal{D}$-edge is of size at least 2 , and moreover no $\mathcal{C}$-edge $(\mathcal{D}$-edge) is included in another $\mathcal{C}$-edge $(\mathcal{D}$-edge $)$. It follows from the splitting-contraction algorithm $[12,13]$ that the 
colouring properties of an arbitrary mixed hypergraph may be obtained from its reduced mixed hypergraph. Therefore, unless otherwise stated, we consider only reduced mixed hypergraphs.

If $\mathcal{H}=(V, \mathcal{C}, \mathcal{D})$ is a mixed hypergraph, then the subhypergraph induced by $V^{\prime} \subseteq V$ is the mixed hypergraph $\mathcal{H}^{\prime}=\left(V^{\prime}, \mathcal{C}^{\prime}, \mathcal{D}^{\prime}\right)$ defined by setting $\mathcal{C}^{\prime}=\left\{C \in \mathcal{C}: C \subseteq V^{\prime}\right\}$, $\mathcal{D}^{\prime}=\left\{D \in \mathcal{D}: D \subseteq V^{\prime}\right\}$ and denoted by $\mathcal{H}^{\prime}=\mathcal{H} / V^{\prime}$.

A mixed hypergraph $\mathcal{H}=(V, \emptyset, \mathcal{D})(\mathcal{H}=(V, \mathcal{C}, \emptyset))$ is called a " $\mathcal{D}$-hypergraph" ("C hypergraph") and denoted by $\mathcal{H}_{\mathcal{D}}\left(\mathcal{H}_{\mathcal{C}}\right)$. If $\mathcal{H}_{\mathcal{D}}$ contains only $\mathcal{D}$-edges of size 2 then it coincides with the usual notion of a graph. A mixed hypergraph $\mathcal{H}=(V, \mathcal{C}, \mathcal{D})$ is called a bi-hypergraph iff $\mathcal{C}=\mathcal{D}$. A subset of vertices which is both a $\mathcal{C}$-edge and $\mathcal{D}$-edge is called a bi-edge.

The study of mixed hypergraphs has made a lot of progress since its inception [13]. It has many potential applications, since mixed hypergraphs can be used to encode various partitioning constraints. They have been used to model problems in such areas as colouring of block designs [3, 4], list-colouring of graphs [10], integer programming [10] and other areas. In this paper we begin a systematic study of planar mixed hypergraphs.

\section{Planar hypergraphs}

Let $\mathcal{H}=(V, \mathcal{E})$ be a hypergraph.

Definition 1 The bipartite representation of $\mathcal{H}$, denoted by $B(\mathcal{H})$, is a bipartite graph with vertex set $V \cup \mathcal{E} . v \in V$ is adjacent to $E \in \mathcal{E}$ (in $B(\mathcal{H})$ ) if and only if $v \in E$.

The following definition is due to Zykov [16]:

Definition 2 A hypergraph $\mathcal{H}$ is called planar iff $B(\mathcal{H})$ is a planar graph.

Thus planar graphs are the special case of planar hypergraphs in which all edges have size 2. As one may see, a planar hypergraph admits an embedding on the plane in such a way that each vertex corresponds to a point on the plane, and every edge corresponds to a closed region homeomorphic to a disk such that it contains the points corresponding to its vertices in the boundary and it does not contain the points corresponding to the other vertices. Furthermore two such regions intersect exactly in the points that correspond to the vertices in the intersection of the corresponding edges. In this way the connected regions of the plane which do not correspond to the edges form the faces of the embedding of the planar hypergraph.

Using properties of the bipartite representation $B(\mathcal{H})$ one can derive many properties of a planar embedding of the hypergraph $\mathcal{H}[1,2,16]$. For example if the degree of vertex $v \in V$ in $\mathcal{H}$ is denoted by $d_{\mathcal{H}}(v)$ we obtain 
Theorem 1 (Euler's formula) If $\mathcal{H}=(V, \mathcal{E})$ is a planar hypergraph embedded on the plane with $f$ faces, then

$$
|V|+|\mathcal{E}|-\sum_{E \in \mathcal{E}}|E|+f=|V|+|\mathcal{E}|-\sum_{v \in V} d_{\mathcal{H}}(v)+f=2 .
$$

Proof. This follows from Euler's formula for $B(\mathcal{H})$.

Definition 3 An embedding of a planar hypergraph is called maximal iff every face contains precisely two vertices, or equivalently iff in the corresponding embedding of $B(\mathcal{H})$ every face has length 4 .

This maximality is relative in the sense that in every such face one can always insert a new edge of size 2. However if a planar hypergraph $\mathcal{H}$ is not maximal then there is at least one face of size at least 3 and therefore one can insert a new edge of size at least 3 in that face.

If we draw the faces of a maximal planar hypergraph as curves connecting respective vertices then we obtain a plane graph whose faces correspond to the edges of the initial hypergraph. In this way, we may look at a plane graph as a planar embedding of a maximal hypergraph such that the faces of the graph correspond to the edges of the hypergraph.

\section{Colouring planar mixed hypergraphs}

Let $\mathcal{H}=(V, \mathcal{C}, \mathcal{D})$ be a mixed hypergraph. Denote the underlying edge-set of $\mathcal{H}$ by $\mathcal{E}=\mathcal{C} \cup \mathcal{D}$. Observe that if some $\mathcal{C}$-edge coincides as a subset of vertices with some $\mathcal{D}$-edge (i.e. it is a bi-edge) then it appears only once in $\mathcal{E}$. We say that $\mathcal{H}^{\prime}=(V, \mathcal{E})$ is the underlying hypergraph of $\mathcal{H}$.

Definition 4 We call a mixed hypergraph $\mathcal{H}=(V, \mathcal{C}, \mathcal{D})$ planar if and only if the underlying hypergraph $\mathcal{H}^{\prime}$ is planar.

This can be viewed as follows: we can embed $\mathcal{H}^{\prime}$ in the plane and label all hyperedges with $b, c$ or $d$ appropriately according to whether they are bi-edges, $\mathcal{C}$-edges or $\mathcal{D}$-edges. Note that $\mathcal{C}$-edges of size 2 can be contracted, and bi-edges of size 2 lead to uncolourability, so that in general it suffices to only consider mixed hypergraphs containing neither.

Example 1 Clearly not every mixed hypergraph is planar. Let $\mathcal{H}_{7}$ be the 3-uniform hypergraph with vertex-set $V\left(\mathcal{H}_{7}\right)=\mathbb{Z}_{7}$, and edge-set $\mathcal{E}\left(\mathcal{H}_{7}\right)=\{\{0+i, 1+i, 3+i\},\{0+i, 2+i$, In $\mathcal{H}_{7}$ every pair of vertices appears in exactly 2 edges, but $B\left(\mathcal{H}_{7}\right)$ has 21 vertices, 42 edges and girth 4 , and is therefore not planar. It can also be proved by exhaustion in a few tedious minutes that $\mathcal{H}_{7}$ is uncolourable as a bi-hypergraph. It is worth noting that $\mathcal{H}_{7}$ is embeddable in the torus: The faces of the standard embedding of $K_{7}$ on the torus, shown 
THE EleCtronic Journal of COMBinatorics 7 (2000), \#R60

in Figure 1, are precisely $\mathcal{E}\left(\mathcal{H}_{7}\right)$. We note also that the edges of this hypergraph are the blocks of the unique simple $S(7,3,2)$ design. The study of designs as mixed hypergraphs is also an important emerging subfield [3].

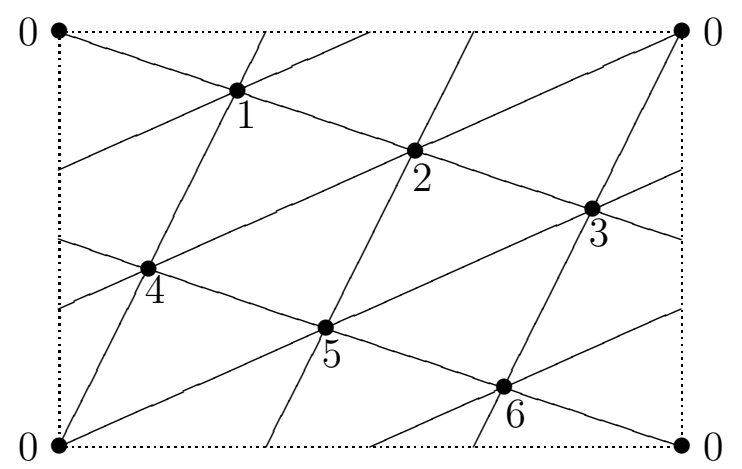

Figure 1: An embedding of $K_{7}$ on the torus

A first discussion on the colouring of planar hypergraphs can be found in a paper of Zykov [16]. The main results discussed there may be reformulated in the language of mixed hypergraphs as follows.

Theorem 2 (Bulitco) The Four colour theorems for planar graphs and for planar $\mathcal{D}$ hypergraphs are equivalent.

Theorem 3 (Burshtein, Kostochka) If a planar $\mathcal{D}$-hypergraph contains at most one $\mathcal{D}$-edge of size 2 then $\chi(\mathcal{H}) \leq 2$.

The question of colouring properties of general planar mixed hypergraphs was first raised in [13] (problem 8, p.43). It is evident that every planar $\mathcal{D}$-hypergraph is colourable, just as every planar $\mathcal{C}$-hypergraph is. The situation changes however if we consider general planar mixed hypergraphs. The smallest non-trivial (reduced) uncolourable planar mixed hypergraph $\mathcal{H}=(V, \mathcal{C}, \mathcal{D})$ is given by $V=\{1,2,3\}, \mathcal{C}=\{(1,2,3)\}, \mathcal{D}=$ $\{(1,2),(2,3),(1,3)\}$. It is easy to embed it on the plane with 4 faces (3 containing 2 vertices each and 1 containing 3 vertices). It is not difficult to extend this example to an infinite family of uncolourable planar mixed hypergraphs. Take for example an odd $\mathcal{D}$-cycle of length $2 k+1$ and add to it $k$ triples of the form $(1,2,3),(3,4,5),(5,6,7), \ldots$ as $\mathcal{C}$-edges, see [14]. The general structure of uncolourable planar mixed hypergraphs is unknown, but we will see that the presence of $\mathcal{D}$-edges of size 2 is crucial. In general $\mathcal{D}$-edges of size 2 are problematic, since allowing them implies that the four colour problem is a special case of the theory of planar mixed hypergraphs.

Since we already excluded $\mathcal{C}$-edges and bi-edges of size 2 , it seems reasonable to study only planar mixed hypergraphs without edges of size 2 . The first interesting case is that in which $\mathcal{H}=(V, \mathcal{C}, \mathcal{D})$ is a maximal 3 -uniform planar bi-hypergraph. These graphs already have a rich structure that will allow us to draw interesting conclusions for the general case, and we will study them in the next section. 


\section{Bi-triangulations}

We now consider the colouring problem for maximal 3-uniform planar bi-hypergraphs $\mathcal{H}$. Since every face of a maximal planar hypergraph is of size 2 , we can associate a graph $G(\mathcal{H})$, on the same vertex set, with $\mathcal{H}$ : replace every face in $\mathcal{H}$ by an edge in $G$, so that every edge in $\mathcal{H}$ becomes a face of $G$. $\mathcal{H}$ is maximal 3 -uniform, so that $G$ must be a triangulation in the usual sense. We use $\mathcal{H}$ and $G$ interchangeably, and since every edge of $\mathcal{H}$ is a bi-edge, we will refer to them as bi-triangulations.

In this section we will study the colourings of bi-triangulations: we want to colour $V(G)$ so that every face has exactly two vertices of the same colour.

Definition 5 A colouring $c_{1}$ is a refinement of a colouring $c_{2}$ if every colour class of $c_{1}$ is contained in a colour class of $c_{2}$. A colour class of a colouring $c$ is maximal if it is a colour class in every refinement of $c$. If every colour class of $c$ is maximal, then $c$ is a maximal colouring.

Lemma 4 A colour class of a colouring of a bi-triangulation is maximal if and only if it induces a connected subgraph.

Proof. In a triangulation two vertices are together in a face if and only they are adjacent. Therefore a maximal colour class must induce a connected subgraph, since otherwise we can simply recolour one of the components. Conversely, a connected colour class can not be refined furthermore, since recolouring some of its vertices would result in two adjacent vertices from the old colour class receiving distinct new colours. This leads to a contradiction, since any face containing these two vertices is now multicoloured.

Observe that this result does not extend to general planar graphs, as can be easily seen by colouring $C_{4}$ with two colours, so that every vertex is adjacent to a vertex of the same colour. The following results (Theorem 5 and Corollaries 1 and 2) also break down for $C_{4}$.

Theorem 5 There is a 1-1 correspondence between the $k$-colourings of a bi-triangulation $G$ and the $k$-face-colourings of the 2-factors in the dual $G^{*}$. In this correspondence a colouring $c_{1}$ of $G$ is a refinement of a colouring $c_{2}$ if and only if the corresponding 2-factors are identical and the face-colouring associated with $c_{1}$ is a refinement of the face-colouring associated with $c_{2}$.

The main idea of the proof is due to Penaud [7] who essentially showed that there is a 1-1 correspondence between 2-colourings of $G$ and 2-factors of $G^{*}$. (See Corollary 2) Proof. A 2-factor of $G^{*}$ partitions the plane into regions, inducing a partition of $V(G)$ into non-empty sets. Thus every proper face-colouring of this 2-factor with $k$ colours corresponds to a $k$-colouring of $V(G)$. Such a colouring is in fact a $k$-colouring of the bi-triangulation $G$, since it follows from the properness of the face-colouring that in every face of $G$ there are exactly 2 vertices from one colour class and 1 from another. 
Conversely, given a $k$-colouring we can recover the 2 -factor and its face-colouring. Since in every face of $G$ there are exactly 2 vertices of the same colour we get a 2-regular spanning subgraph, i.e. a 2-factor, of $G^{*}$ by taking the dual edge of every edge in $G$ that is incident to vertices of different colour. Now, if two vertices are in the same region (generated by the 2-factor) then there is a curve connecting them that passes only through vertices in this region. But then consecutive vertices on this curve must be on the same face and are therefore adjacent. The edge joining these vertices can not be the dual of an edge in the 2-factor, since otherwise it would follow from the Jordan curve theorem that they are in different faces. By the definition of the 2-factor it follows thus that consecutive vertices on this curve must be of the same colour, and that therefore every vertex in a given region has the same colour. Since every region of the 2-factor must contain at least one vertex we can therefore uniquely define the colouring of the regions and this $k$-colouring is a good-colouring since faces are separated by dual edges and thus adjacent faces contain adjacent vertices of different colour.

For the second part of the proof observe that a refinement of the face-colouring of the dual graph clearly leads to a refinement of the colouring of the bi-triangulation. For the converse suppose that $c_{1}$ is a refinement of $c_{2}$. Following the construction of the dual 2 -factor it follows that the 2-factor for $c_{1}$ must contain the 2-factor for $c_{2}$, from which it follows that they are identical. It follows that the face-colouring corresponding to $c_{1}$ must be a refinement of the colouring for $c_{2}$.

Definition 6 Recall that $r_{k}(G)$ is the number of different strict $k$-colourings of $G$ (disregarding permutations of colors), i.e. the number of partitions of $V(G)$ into $k$ non-empty sets that describe a good-colouring of the mixed hypergraph $G$. Let $S(n, k)$ denote the Stirling numbers of the second kind, i.e. the number of ways of partitioning a set of $n$ elements into exactly $k$ sets. Also define $f_{k}\left(G^{*}\right)$ to be the number of 2-factors of $G^{*}$ that consist of exactly $k$ components, and let $f\left(G^{*}\right)=\sum_{i \geq 1} f_{i}\left(G^{*}\right)$ be the total number of 2-factors of $G^{*}$.

Corollary 1 Every colouring of a bi-triangulation $G$ can be refined to a unique maximal colouring. Furthermore, there are exactly $f_{k-1}\left(G^{*}\right)$ maximal $k$-colourings of $G$.

Proof. By the Jordan curve theorem a given 2-factor consisting of $k-1$ cycles divides the plane into $k$ regions and by Lemma 4 the colouring that assigns a different colour to each face must be the unique maximal colouring for this 2-factor, since (as it was shown in the proof above) the vertices in every region induce a connected subgraph. The second statement follows immediately. All refinements of a given colouring correspond to the same 2-factor, so that the first statement also follows.

Corollary 2 A bi-triangulation $G$ has exactly $2 f\left(G^{*}\right)$ proper 2-colourings. In general,

$$
r_{k}(G)=\sum_{i \geq 1} S(i, k-1) f_{i}\left(G^{*}\right), \text { and }
$$




$$
P(\mathcal{H}, \lambda)=\sum_{i \geq 1} f_{i}\left(G^{*}\right) \lambda(\lambda-1)^{i}
$$

Proof. The first statement follows from both summation formulas, by setting $k=2$ or $\lambda=2$ respectively. For the first formula it suffices by Theorem 5 to show that every 2 -factor consisting of $i$ cycles can be $k$-face-coloured in exactly $S(i, k-1)$ ways. To see this, create a graph whose vertex-set are the faces in the dual of the 2 -factor, and two vertices are adjacent if and only if the corresponding faces are separated by a 2-factor. This graph is connected and has $i$ edges. By the Jordan curve theorem it has exactly $i+1$ vertices and must therefore form a tree $T$. Let $r_{k}(T)$ be the number of proper $k$-colourings of $T$. To see that $r_{k}(T)=S(e(T), k-1)$, observe that $r_{1}\left(K_{1}\right)=1$ and $r_{k}\left(K_{1}\right)=0$ for $k \geq 2$. By removing a leaf $v$ we can see that $r_{k}(T)=(k-1) r_{k}(T-v)+r_{k-1}(T-v)$, the usual recursion for the Stirling numbers. For the second formula it now suffices to observe that the chromatic polynomial for a tree on $i+1$ vertices is $\lambda(\lambda-1)^{i}$.

Jiang, Mubayi, Tuza, Voloshin and West [6] have exhibited mixed hypergraphs whose chromatic spectrum is not an interval, i.e. there may be some zeroes between positive components. This can not happen for bi-triangulations.

Corollary 3 The spectrum of every bi-triangulation $G$ is unbroken, $\chi(G)=2$ and $\bar{\chi}(G)=$ $1+\max \left\{k: f_{k}\left(G^{*}\right) \geq 1\right\}$.

Proof. Since $G^{*}$ is a 3-regular bridgeless graph it follows from Petersen's theorem that it has a 2-factor. So by Corollary 2 every bi-triangulation is 2-colourable, and therefore must have lower chromatic number 2. A colouring achieving the upper chromatic number must be maximal, so that the value of $\bar{\chi}(G)$ follows from Corollary 1. If $k=\bar{\chi}(G)$, then $f_{k-1}\left(G^{*}\right) \geq 1$, so since $S(k-1, i-1) \geq 1$ for every $2 \leq i \leq k$, we get that $r_{i}(G) \geq 1$ in this range and the spectrum is unbroken. Furthermore an $i$-colouring can be obtained from an $i$-colouring of the tree.

Corollary 4 Every planar mixed hypergraph without edges of size 2 can be 2-coloured.

Proof. Without loss of generality we may assume that the mixed hypergraph is a maximal bi-hypergraph, since adding $\mathcal{C}$ - or $\mathcal{D}$-edges only decreases the number of 2 -colourings. Similarly, if $G$ contains any faces of size larger than 3, then those can be divided into faces of size 3 by adding graph edges to obtain a bi-triangulation. The result now follows from Corollary 3.

Corollary 5 Every uniquely colourable planar mixed hypergraph must have an edge of size 2.

Proof. Suppose that $G$ is uniquely colourable, and free of edges of size 2. Again we may assume that $G$ is a bi-triangulation. By Corollary $3, \bar{\chi}(G)=2$, so that by Corollary 2, $G^{*}$ must have a unique 2-factor: a Hamiltonian cycle. This contradicts the following theorem. 
Theorem 6 (Thomason [8], Tutte [9] "Smith's Theorem") The number of Hamiltonian cycles through a given edge of a cubic graph is even.

Proof. We sketch the elegant proof of Thomason. Let $u v$ be the given edge. Consider the graph whose vertices are the Hamiltonian paths starting at $u$ with edge $u v$. Two such paths are adjacent if one can be obtained from the other by adding an edge at the end of the path and deleting a different edge. Now vertices of degree 1 in this graph correspond to Hamiltonian cycles containing $u v$ and all other vertices have degree 2. Thus the number of Hamiltonian cycles containing $u v$ is even.

Example 2 Consider the bi-triangulation corresponding to $K_{4}$, i.e. $\mathcal{H}=(V, \mathcal{C}, \mathcal{D})$ with $V=\{1,2,3,4\}, \mathcal{C}=\mathcal{D}=\{(123),(234),(341),(412)\}$. It is easy to see that $K_{4}$ is self-dual and that all 2-factors of $K_{4}$ are Hamiltonian. Thus $\chi(\mathcal{H})=\bar{\chi}(\mathcal{H})=2$. One can also verify that the strict colourings with two colours are 1212, 1122, 2112 and the corresponding three 2-factors of $G^{*}$ are 12341, 13241 and 12431, so that the chromatic spectrum is $R(\mathcal{H})=(0,3,0,0)$ and the chromatic polynomial is $3 \lambda(\lambda-1)$.

\section{Bounds for the upper chromatic number}

Recall that the upper chromatic number of a $\mathcal{D}$-hypergraph on $n$ vertices is always $n$. If we have at least one $\mathcal{C}$-edge then we have $\bar{\chi} \leq n-1$. Perhaps surprisingly, this bound can be achieved even for the restricted class of bi-triangulations.

Definition 7 If we replace an edge ab of a graph by creating a new digon cd and including the edges ac and bd, then we say that we are inserting a digon. The new graph is cubic if and only if the original graph was cubic.

Similarly, inserting a $K_{4}-e$ corresponds to removing the edge ab, creating 4 new vertices $\{c, d, e, f\}$ and including the edges $\{a c, b d, c e, c f, d e, d f, e f\}$.

$C_{3}^{*}$ is the 2-vertex graph consisting of a triple-edge.

Example 3 Let $C_{2 n}^{\prime}$ denote the cubic graph on $2 n$ vertices that is obtained by replacing every edge of a perfect matching in $C_{2 n}$ by a double-edge. $C_{2 n}^{\prime}$ can also be built recursively by inserting $n-1$ digons into the same edge of $C_{3}^{*}$. Consider the bi-triangulation on $n$ vertices $\mathcal{H}$, with underlying graph $G$, such that $G^{*}=C_{2 n-4}^{\prime}$. It follows from Corollary 3 that $\bar{\chi}(\mathcal{H})=n-1$, since the $n-2$ digons form a 2-factor in $G^{*}$ with the maximum number of cycles.

It can be seen that this is the unique $n$-vertex bi-triangulation that achieves the bound. We notice that in the example $\mathcal{H}, G$ and $G^{*}$ all have repeated edges, and this is crucial for achieving the bound. For this reason we will regard $\mathcal{E}$ as a multi-set, i.e. we keep track of how many copies there are of each bi-edge. 
Lemma 7 Let $\mathcal{H}$ be the bi-triangulation corresponding to a triangulation $G$ other than $C_{3}$.

1. An edge of $\mathcal{H}$ can be repeated at most twice.

2. If $G^{*}$ has repeated edges, then $G$ has repeated edges.

3. Every pair of vertices in $G^{*}$ that form a digon gives rise to a unique pair of repeated edges in $\mathcal{H}$.

4. $G^{*}$ has repeated edges if and only if $\mathcal{H}$ has repeated edges.

Proof. For 1. consider the edge $a b c$ in $\mathcal{H}$ and suppose that in $G$ the edge $a b$ appears $k$ times. The $k$ copies of $a b$ divide the plane into $k$ regions, only one of which can contain $c$. Thus $a b c$ is repeated at most twice.

For the remaining parts suppose that $a b$ is a repeated edge of $G^{*}$ and let $a x$ and $b y$ be the remaining edges at $a$ and $b$ respectively. If $x=b$, then $y=a$ and $G^{*}$ is a triple-edge, so that $G=C_{3}$. Otherwise $a b$ forms a digon. Because $G^{*}$ is 2 -connected (as the dual of a planar graph) both $x$ and $y$ must be internal or external to the digon, so that the digon forms a face $C$ in $G^{*}$. Furthermore, every face in $G^{*}$ containing ax must also contain a copy of $a b$ and by. There are exactly two such faces $A$ and $B$. So the edges in $G$ that are dual to $a x$ and by must be two copies of the edge $A B$, proving 2 . For 3 . observe that $\{A B C\}$ is a repeated edge of $\mathcal{H}$, once corresponding to $a$ and once to $b$.

For 4. it now suffices to prove the reverse implication, so suppose that $A B C$ appears twice. If $A B$ and $A C$ are not repeated, then the two faces $A B C$ are connected by exactly two edges $B C$ and $A C$, forming a digon in $G^{*}$.

The reverse implications in Lemma 7.2/3 need not hold in general.

Example 4 Let $C_{2 n}^{\prime \prime}$ be the cubic graph on $4 n$ vertices obtained by inserting $n-1$ copies of $K_{4}-e$ into the same edge of $K_{4}$. If we again let $G^{*}=C_{2 n}^{\prime \prime}$, then $G^{*}$ and $\mathcal{H}$ have no repeated edges, but $G$ forms a bi-triangulation with multiple edges.

Example 5 Let $\Theta^{\prime}$ be the cubic graph on 8 vertices obtained by inserting a digon into every edge of $C_{3}^{*}$. So $\Theta^{\prime}$ has 3 digons, but the corresponding hypergraph contains 4 pairs of identical hyperedges.

Theorem 8 If $\mathcal{H}$ is a bi-triangulation with $n$ vertices and $m$ repeated edges, then $\bar{\chi}(\mathcal{H}) \leq$ $\lfloor(2 n+m-1) / 3\rfloor$ and this bound is sharp.

Proof. Let $\mathcal{H}$ have $G$ as its underlying graph, and suppose that $G^{*}$ contains $d$ digons. We first notice that the digons are vertex-disjoint (since $G^{*}$ is cubic) and by Lemma 7.3 we get $d \leq m$. In a 2 -factor of $G^{*}$ with the maximum number of cycles every vertex not 
in a digon must be in a cycle of length at least 3 . Thus, since $G^{*}$ has exactly $n^{*}=2 n-4$ vertices, such a 2 -factor has at most $d+\left(n^{*}-2 d\right) / 3$ cycles and therefore:

$$
\bar{\chi}(\mathcal{H}) \leq 1+d+\left\lfloor\frac{2 n-4-2 d}{3}\right\rfloor=\left\lfloor\frac{2 n+d-1}{3}\right\rfloor \leq\left\lfloor\frac{2 n+m-1}{3}\right\rfloor .
$$

We shall now prove that the upper bound is sharp by constructing the appropriate cubic planar graph $G^{*}$. In a $Y-\Delta$ transformation of a cubic graph a vertex $v$ with neighbors $w_{1}, w_{2}, w_{3}$ is replaced by a triangle $v_{1} v_{2} v_{3}$ as follows: Remove $v$, and introduce new vertices $v_{1}, v_{2}, v_{3}$ and the edges $\left\{v_{1} v_{2}, v_{1} v_{3}, v_{2} v_{3}, v_{1} w_{1}, v_{2} w_{2}, v_{3} w_{3}\right\}$. The new graph is still cubic planar and there are no multiple edges involving the new vertices.

Let $2 n-4-2 m=3 t+r$ with $0 \leq r \leq 2$. Observe that $t-r$ must be even and suppose that $t>r$, so that $C_{t-r}^{\prime}$ is a cubic planar graph. Perform a $Y-\Delta$ transformation at every vertex in $C_{t-r}^{\prime}$ to obtain a simple cubic planar graph on $3 t-3 r$ vertices that contains exactly $t-r$ disjoint triangles. Next pick an edge $u v$ that is not contained in a triangle and insert $r K_{4}-e$ 's into $u v$. We now have a graph $G_{0}$ on $3 t+r$ vertices which contains a 2-factor consisting of $t$ cycles. If $0<t \leq r$, then $3 t+r \in\{4,8\}$ and we let $G_{0}$ be $K_{4}$ or the graph obtained from $K_{4}$ by inserting a $K_{4}-e$ into some edge $u v$. In either case $G_{0}$ contains no pair of vertices incident to the same faces. Now obtain $G^{*}$ by inserting $m$ digons into $u v$, and if $t=0=r$ we let $G^{*}=C_{2 m}^{\prime}$. Hence $G^{*}$ is a cubic, planar graph on $2 n-4$ vertices and has a 2 -factor consisting of $t+m$ cycles. The corresponding bi-triangulation $\mathcal{H}$ has $n$ vertices, $m$ repeated edges, and

$$
\bar{\chi}(\mathcal{H}) \geq t+m+1=\left\lfloor\frac{2 n+m-1}{3}\right\rfloor .
$$

In the remaining case when $3 t+r=2$ we must have $m=n-3$ and all but exactly 2 faces are repeated. Observe that no face may have a repeated edge, since then the third edge of the face would have to be a loop. However, since every edge must be in an even number of faces and all but 2 faces are repeated, every edge that occurs in one of these

2 faces must also occur in the other. But then these faces would have to be identical, a contradiction, so that this case can't occur.

\section{Open Problems}

We raise some open questions in this section.

1. For which planar bi-hypergraphs is $\bar{\chi}(G)=\chi(G)$ ? or even

2. Is $K_{4}$ the only bi-triangulation with $\bar{\chi}(G)=2$ ?

3. For which bi-hypergraphs of given genus $\mathrm{g}$ is $\bar{\chi}(G)=\chi(G)$ ?

4. Is it NP-hard to determine the upper chromatic number of a bi-triangulation? 
THE EleCtronic Journal of COMBinatorics 7 (2000), \#R60

5. Are there bi-hypergraphs/planar mixed hypergraphs (without edges of size 2) for which the spectrum is broken? or more generally

6. What is the smallest genus of a mixed hypergraph that has gaps in its chromatic spectrum?

REMARKS: Question 2 is difficult, since the equivalent question of "Is $K_{4}$ the unique cubic planar graphs all of whose 2-factors are Hamiltonian cycles?" has been investigated with only partial success and counter-examples can be found if one drops the assumption of planarity [5].

The hypergraph $(\{a, b, c\},\{a b c, a b c, a b c\})$ is embeddable in the torus as its incidence graph is $K_{3,3}$ and one trivially has $\bar{\chi}(G)=\chi(G)=2$.

Simply examining all 2-factors of the dual is not a successful strategy for question 4, since for example the double-wheel $\bar{K}_{2} \vee C_{n}$ has upper chromatic number $1+\lfloor n / 2\rfloor$, but

$F_{n}+F_{n-2}+2(n+1 \bmod 2) \in \Theta\left(\left(\frac{1+\sqrt{5}}{2}\right)^{\mathrm{n}}\right)$ 2-colourings (and accordingly 2-factors in the dual), where $F_{n}$ is the $n$-th Fibonacci number. This follows, since there are exactly two 2-colourings in which the center vertices have different colour when $n$ is even, none otherwise, and the number of colourings with both vertices having the same colour is equal to the number of independent sets in $C_{n}$, i.e. $F_{n}+F_{n-2}$.

\section{Acknowledgements}

The authors would like to thank Radhika Ramamurthi, and Kathie Cameron for useful suggestions and fruitful discussions.

\section{References}

[1] C. Berge, Graphs and Hypergraphs, North Holland, 1973.

[2] C. Berge, Hypergraphs: Combinatorics of finite sets, North Holland, 1989.

[3] C. Colbourn, J. Dinitz and A. Rosa, Bicoloring Triple Systems Electronic J. Combin. 6\# 1, paper 25, 16 pages (electronic).

[4] Ch.J. Colbourn and A. Rosa, Triple Systems. Clarendon Press, Oxford, 1999 (sect.18.6. Strict colourings and the upper chromatic number, p.340-341).

[5] M. Funk, B. Jackson, D. Labbate and J. Sheehan, 2-Factor Hamilton Graphs, preprint.

[6] T. Jiang, D. Mubayi, Zs. Tuza, V. Voloshin and D.B. West, Chromatic spectrum is broken. 6th Twente Workshop on Graphs and Combinatorial Optimization, 26-28 May, 1999, H.J. Broersma, U.Faigle and J.L. Hurink (eds.). University of Twente, May, 1999, 231-234. 
THE ElECtronic Journal of COMBinatorics 7 (2000), \#R60

[7] J.G. Penaud, Une propriété de bicoloration des hypergraphes planaires. (French) Colloque sur la Théorie des Graphes (Paris, 1974) Cahiers Centre Études Recherche Opér. 17 (1975), 345-349.

[8] A.G. Thomason, Hamiltonian cycles and uniquely edge colourable graphs, Annals of Discrete Math. 3 (1978), 259-268.

[9] W.T. Tutte, On Hamiltonian circuits J. London Math. Soc. 21 (1946), 98-101.

[10] Zs. Tuza and V. Voloshin, Uncolourable mixed hypergraphs, Discrete Appl. Math. 99 (2000), 209-227.

[11] Zs. Tuza, V. Voloshin and H. Zhou, Uniquely colourable mixed hypergraphs, submitted.

[12] V. Voloshin, The mixed hypergraphs, Computer Science Journal of Moldova 1 (1993), $45-52$.

[13] V. Voloshin, On the upper chromatic number of a hypergraph, Australasian Journal of Combinatorics 11 (1995), 25-45.

[14] V. Voloshin and H.-J. Voss, Circular Mixed Hypergraphs I: colourability and unique colourability, Congressus Numerantium, 142-147 2000, in print.

[15] D.B. West, Introduction to Graph Theory. Prentice Hall, 1996.

[16] A.A. Zykov, Hypergraphs (in Russian), Uspekhi Mat. Nauk 29 (1974), 89-154. 